\title{
Research Paper \\ Psychometric Characteristics of Persian Version of Parenting Style Index
}

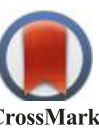

Mohammad Mehrad Sadr ${ }^{1}$, Noushin Khademolreza², Saharnaz Akhbari ${ }^{3}$, Marziyeh Olamaei ${ }^{1}$, *Seyed Sepehr Hashemian ${ }^{1}$

1. PhD Candidate, Department of Clinical Psychology, Faculty of Psychology and Educational Sciences, Allameh Tabataba'i University, Tehran, Iran.

2. Psychiatrist, Assistant Professor, Department of Psychiatry, School of Behavioral Sciences and Mental Health (Tehran Institute of Psychiatry), Iran University of Medical Sciences, Tehran, Iran

3. MSc., Department of Clinical Psychology, Faculty of Psychology and Educational Sciences, Allameh Tabataba'i University, Tehran, Iran.

\begin{tabular}{|c|c|}
\hline $\begin{array}{l}\text { Use vour device to scan } \\
\text { and read the article online }\end{array}$ & retertsons \\
\hline 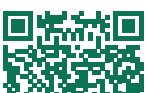 & $\begin{array}{l}\text { Citation: Sadr MM, Khademolreza N, Akhbari S, Olamaei M, Hashemian SS. [Psychometric Characteristics of Persian } \\
\text { Version of Parenting Style Index (Persian)]. Iranian Journal of Psychiatry and Clinical Psychology. 2018; 24(1):80-91. https://doi. } \\
\text { org/10.29252/NIRP.IJPCP.24.1.80 }\end{array}$ \\
\hline 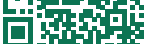 & d oli": https://doi.org/10.29252/NIRP.IJPCP.24.1.80 \\
\hline
\end{tabular}

Received: 02 Jan. 2017

Accepted: $23 \mathrm{Jul} .2017$

Key words:

Factor structure, Reliability, Parenting style, Parenting style index

\begin{abstract}
A B S T R A C T
Objectives The present study investigated the factor structure and reliability of the Parenting Style Index 2. Methods A total of 381 students of Hakim Sabzevari University filled out the survey. An exploratory and confirmatory factor analysis was used to investigate the factor structure of the method, and Cronbach's alpha and test-retest methods were employed for examining the reliability.

Results The results of the principal component with varimax rotation showed three- and four-factorial solutions that explained $45.13 \%$ and $52.17 \%$ of the total variance, respectively. The results of the confirmatory factor analysis showed that the three-factorial solution was a better indication of the data than the four-factorial solution and that it fitted the data adequately in the Iranian society. The Cronbach's alpha of the instrument was 0.65 and that for the subscales of the instrument was $0.53-0.75$. The correlation coefficient of test-retest was 0.77 , which demonstrated acceptable reliability of the instrument. Conclusion Acceptable reliability and fitting well with the data in Iranian society rendered the 3 factor PSI II as a suitable measure for determining the perceived parenting style with respect to screening or clinical purposes.
\end{abstract}

\section{Extended Abstract}

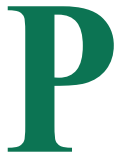

\section{Introduction}

arenting as defined by Bradley and Caldwell is regulating the behavior and development of children with the intention to allow them to have a decent social life, adapt to the environment, and pursue their goals. According to Baumrind, one of the pioneers in the study of parenting style, socializing the child based on the wishes of the community along with retaining the sense of individual integrity is a key element of the role of parent- ing. The classification of parenting style that is most widely used among investigators and researchers was introduced by Martin, based on the work of Baumrind.

They categorized the parenting styles based on two dimensions: Responsiveness (warmth) and demandingness (control). Responsiveness was characterized by kindness, acceptance, and care, whereas demandingness was characterized by limitation, interference, and ordering. The interaction of these two dimensions created four types of parenting styles: authoritative parenting style (high scores in both demandingness and responsiveness), authoritarian parenting style

* Corresponding Author:

Seyed Sepehr Hashemian, PhD Candidate

Address: Department of Clinical Psychology, Faculty of Psychology and Educational Sciences, Allameh Tabataba'i University, Tehran, Iran.

Tel: +98 (915) 5241766

E-mail: sepehr.hashemian@gmail.com 
(high scores in demandingness and low in responsiveness), indulgent parenting style (high scores in responsiveness and low in demandingness), and neglectful parenting style (low scores in both demandingness and responsiveness).

The research on parenting styles has repeatedly revealed that they play a critical role in the growth of individuals. From a dimensional perspective, the positive developmental achievements of children have constantly been associated with the supply of nurture (warmth and responsiveness), encouragement of independence (democracy and autonomy), and appropriate control. Furthermore, several questionnaires have been developed to measure the parenting styles in order to evaluate its importance, some of which, have been translated into Persian and have been used in Persian studies such as Baumrind Parenting Style Inventory (BPSI), Parental Bonding Instrument (PBI), Parental Authority Questionnaire (PAQ), Parenting Style Dimensions Questionnaire (PSDQ), Perceived parenting Style Questionnaire (PPSQ), perceived parental rearing behavior (s-EMBU), and Parenting Style Questionnaire (PSQ).

However, a questionnaire that measures the four parenting styles identified by Macaque and Martin calls for the opinion of the children about the perceived style. This questionnaire is brief and understandable and has been presented by Darling and Toyokawa, termed as the parenting style questionnaire 2 (PSI II). Parenting Style Questionnaire 1 (PSI I) was designed to measure the parenting style independent of the parenting practice as one of the goals of the questionnaire was to investigate the relationship between the parenting style and the achievement of children in a relatively large age range and population; also, the design of the questionnaire as short, conceptually simple, and reliable survey was preferable. The PSI I questionnaire consisted of three subscales of responsiveness, demandingness, and autonomy granting.

Although the initial reliability tests in high school senior students and university freshman students showed acceptable values, that in the $7^{\text {th }}$ grades students was slightly problematic. Therefore, an overview tool was used to increase the consistency and internal variability of the items while maintaining the conceptual clarity of the structure and the short format. The result was PSI II with two fundamental changes, consisting of additional items that reduced the positive bias in responses and measured the wide range of demandingness structures. Moreover, it also measures similar to a 4-point Likert format, which only harbored positive and negative options with varying degrees changed to a 5-points Likert version such that the neutral response could be selected.

\section{Method}

The present study investigated the factor structure and reliability of PSI II. A total of 381 students of Hakim Sabzevari University filled out the instrument. Exploratory and confirmatory factor analyses were used to investigate the factor structure of the instrument, and Cronbach's alpha and test-retest method were employed for examining the reliability.

\section{Results}

The results of the principal component with varimax rotation revealed 3- and 4-factorial solutions that explained $45.13 \%$ and $52.17 \%$, respectively, of the total variance, respectively. The results of the confirmatory factor analysis showed that the 3-factorial solution was a better indication of the data than the 4-factorial solution and that the 3-factorial solution better fits the data in the Iranian society. The Cronbach's alpha of the instrument was 0.65 and varied from 0.53 to 0.75 for the subscales, while the correlation coefficient of test-retest was 0.77 that showed adequate reliability for the instrument. Thus, adequate reliability and fitting well with the data in the Iranian society render the 3-factor PSI II as a suitable measure for determining the perceived parenting style for screening or clinical purposes.

After performing the exploratory factor analysis, the items of the PRQ II in the present study designated the loadings on different factors. Finally, a new nomenclature regarding the content of the items was laid, which led to the elimination of the autonomy granting factor in the questionnaire replaced by the neglect factor in the standardized questionnaire.

\section{Discussion}

Given the many cultural differences in the field of child-rearing, creating an independent tool from the beginning would be a suitable option. Some limitations of this study included the use of the self-report tool and the population of the study that included only the students, as well as the lack of using similar and different questionnaires for the assessment of convergent and discriminant validity. Thus, the results should be generalized with caution. Furthermore, it is recommended to carry out this study on a variety of other 
samples with age variations and large sample size in order to test the repeatability of the results. Finally, acceptable reliability and fitting well with the data in the Iranian society make the 3 -factor PSI II as a suitable measure for determining the perceived parenting style for screening and usability in research and clinical purposes.

\section{Ethical Considerations}

\section{Funding}

This research did not receive any specific grant from funding agencies in the public, commercial, or not-forprofit sectors.

\section{Conflict of Interest}

The authors declare no conflict of interest. 


\title{
ويثَّى هاى روانسنجى نسخه فارسى يرسشنامه سبكهاى فرزنديرورى
}

\author{
محمد مهراد صدر'، نوشين خادم الرضا'، سحرناز اخبارى'، مرضيه علمائى'، "سيدسيهر هاشميان' \\ 1 - دانشجوى دكترا، كروه روانشئاسى بالينى، دانشكده روانشئاسى و علوم تربيتى، دانشكاه علامه طباطبايى، تهران، ايران.

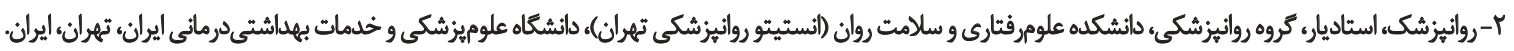

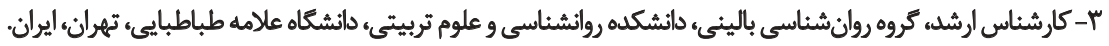

\begin{abstract}
حكيد

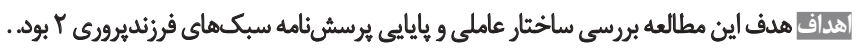

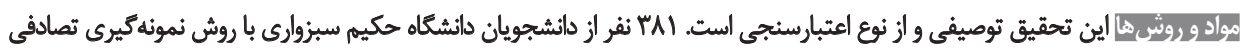

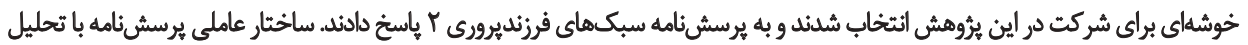

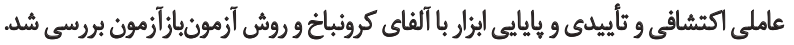

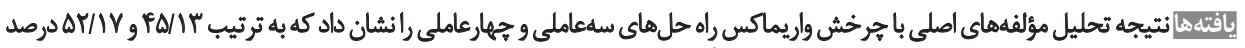

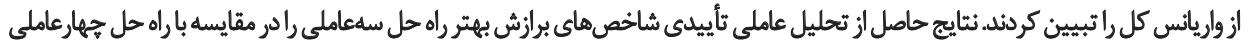

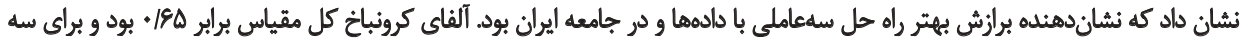

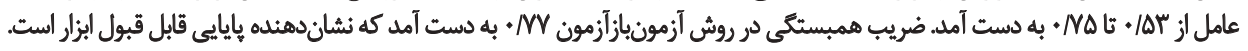

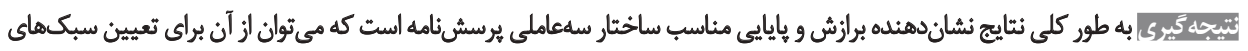

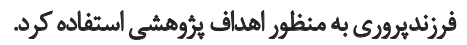

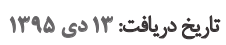

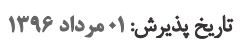

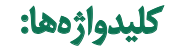
ساختار عاملي، باياييى، سبكهاى فرزنديروي، بائي،

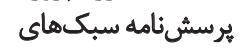

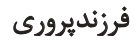

بامريند كه ماكوبى و مارتين "آن را توسعه دادند، كار محققان

dâم

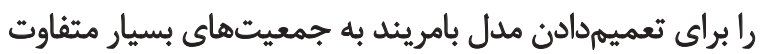

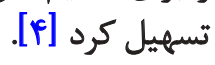

امروزه سبك طبقهبندى انواع فرزنديرورى كه بيشترين و

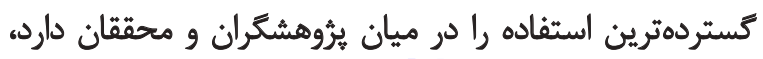

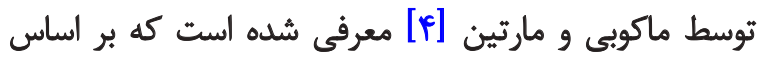

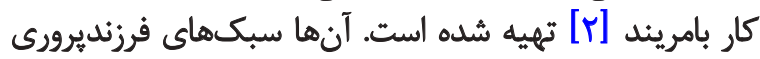

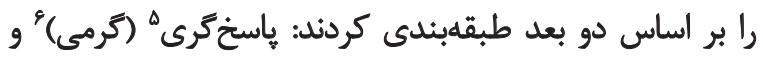

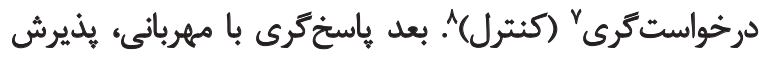

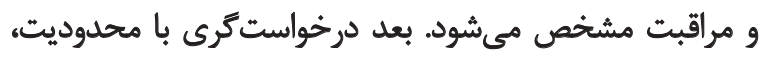

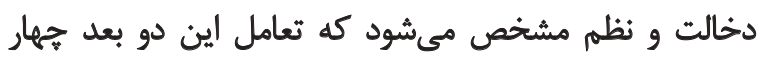

\footnotetext{
4. Maccoby \& Martin

5. Responsiveness

6. Warmth

7. Demandingness

8. Control
}

1. Bradley \& Caldwell

2. Baumrind

3. Permissive

همانَّونه كه بردلى و كالدول ' تعريف كردهاند، فرزنديرورى

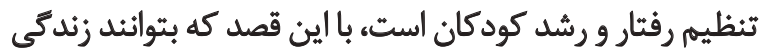

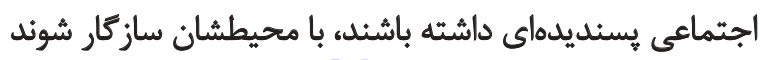

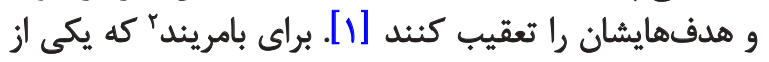

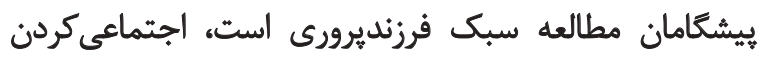

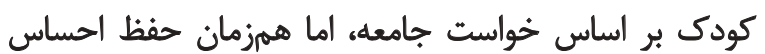

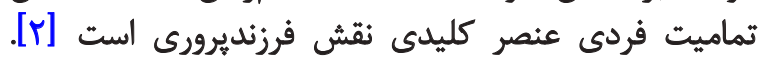

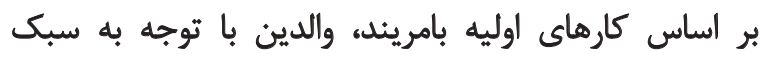

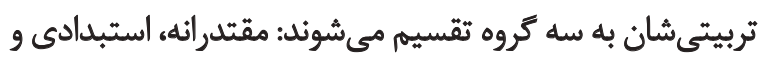

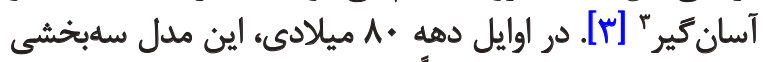

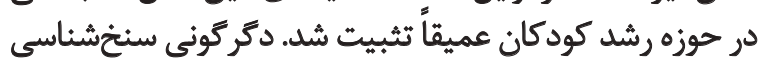

$$
\text { … }
$$

: نويسئده مسينول:

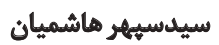
نشانى: تهران، دانشعاه علامه طباطبائ، دانشكله روانشئاسي و علوم تربيتي، كروه روانشئاسى بالينى. تلفئ: sepehr.hashemian@gmail.com يست الكترونيكي 


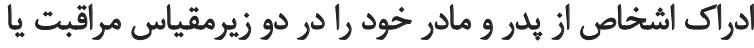

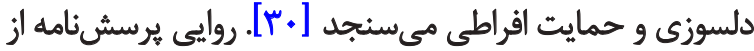

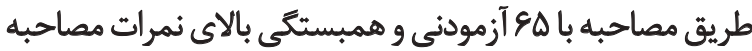

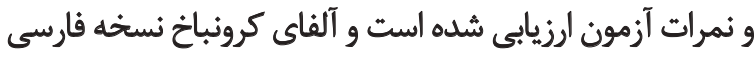

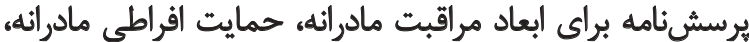

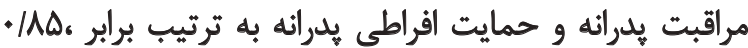

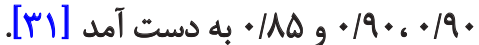

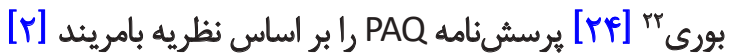

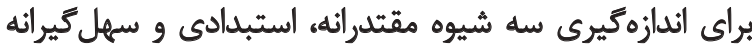

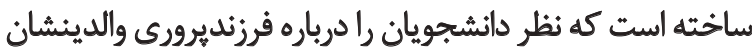

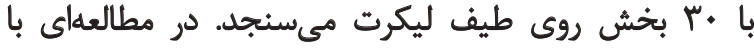

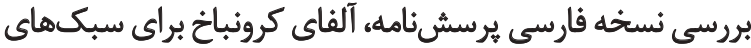

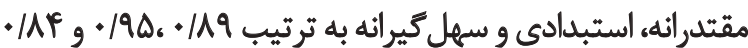

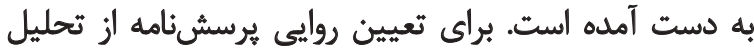

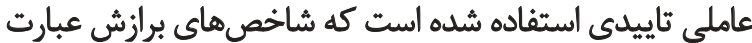

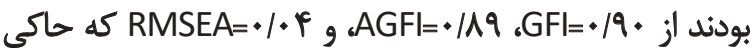

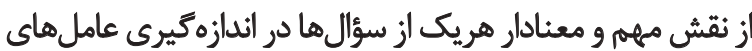

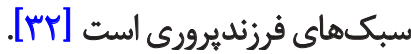

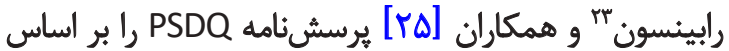

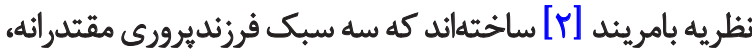

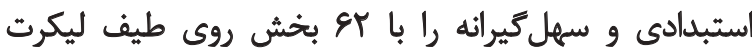

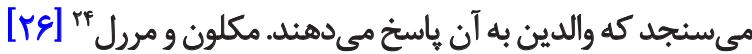

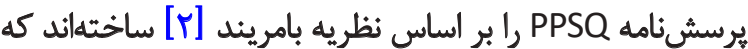

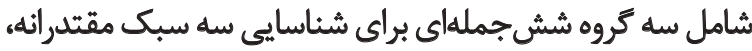

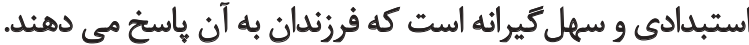

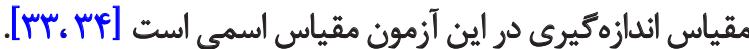

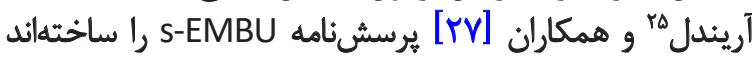

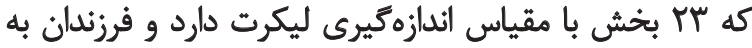

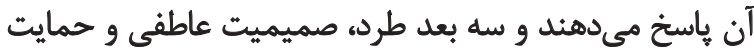

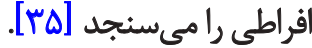

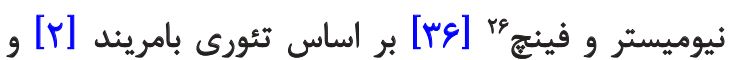

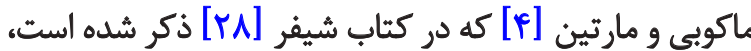

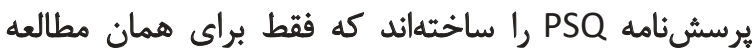

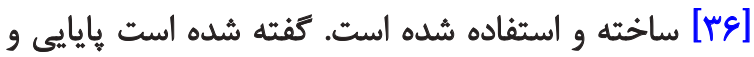

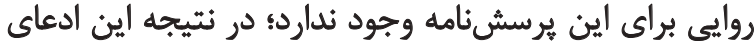

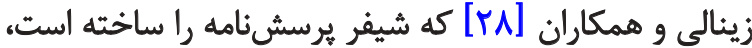

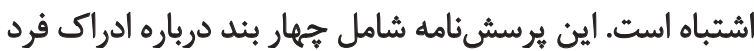

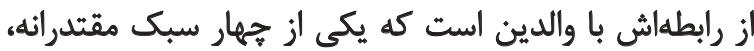

22. Buri

23. Robinson

24. McClun \& Merrell

25. Arrindell

26. Neumeister \& Finch
نوع سبك فرزنديرورى مي سازد: سبك فرزئديرورى مقتدارنه

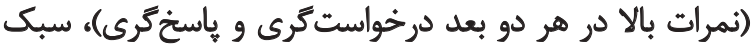

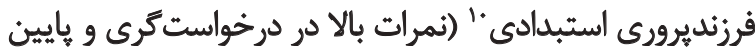

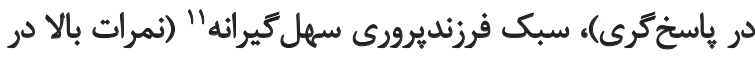
غاسخترى و يايين در درخواست

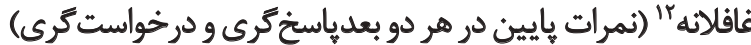

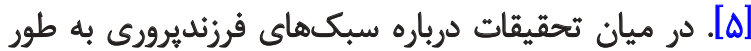

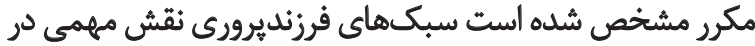

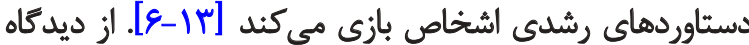

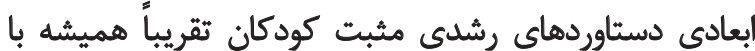

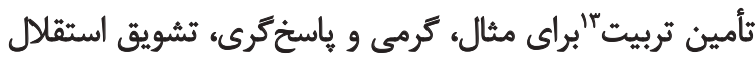

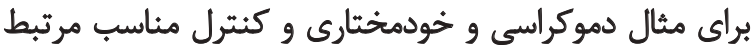

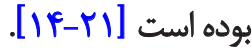

به دليل همين اهميت رشدى سبكهاى فرزنديرورى براى بري يري

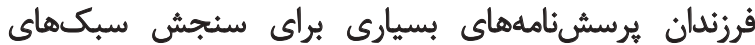

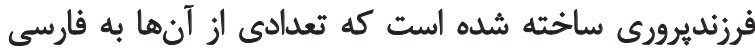
ترجمه شده و در تحقيقات فارسى استفاده شدانداند. از از جمله

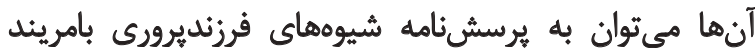

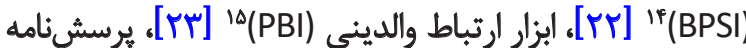

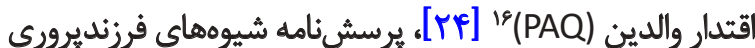

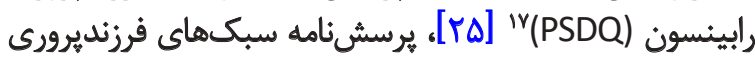

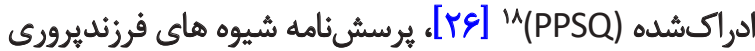

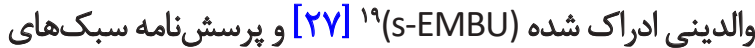

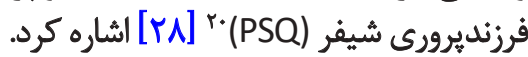

يرسشنامه BPSI اقتباسى است از نظريه اقتدار والدين كه بر إنبا

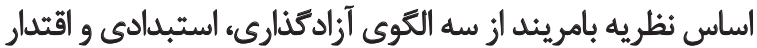

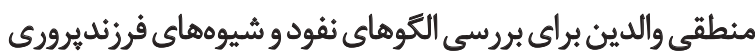

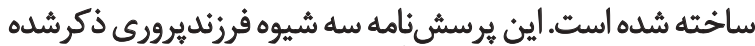

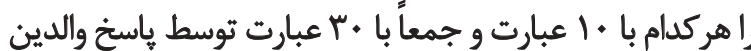

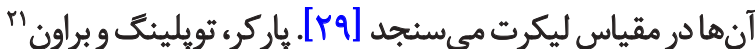

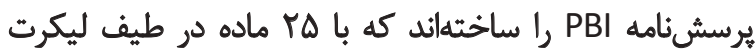

9. Authoritative parenting style

10. Authoritarian parenting style

11. Indulgent parenting style

12. Neglectful parentingstyle

13. Supply of nurture

14. Baumrind Parenting Style Inventory (BPSI)

15. Parental Bonding Instrument (PBI)

16. Parental Authority Questionnaire (PAQ)

17. Parenting Style Dimensions Questionnaire (PSDQ)

18. Perceived parenting Style Questionnaire (PPSQ)

19. Perceived parental rearing behavior (s-EMBU)

20. Parenting Style Questionnaire (PSQ)

21. Parker, Tupling \& Brown 
وضوح مفهومى سازه و فرمت كوتاه آن بود. در نتيجه ارئه إئه

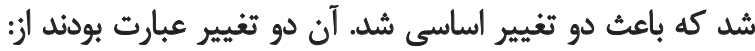

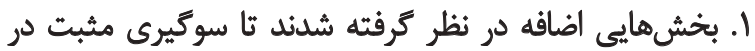

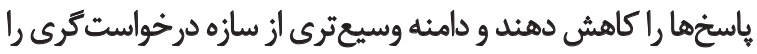

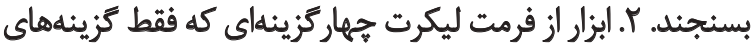

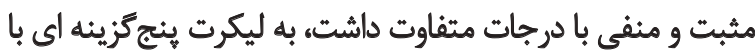
قابليت انتخاب ياسخ خنثى تغيير كرد [rV]

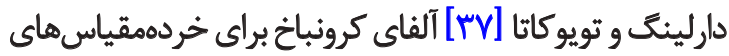

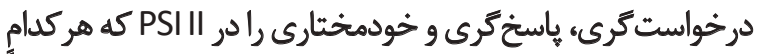

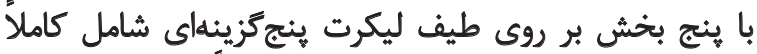

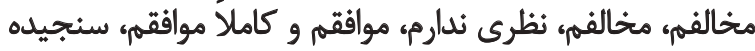

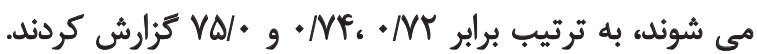

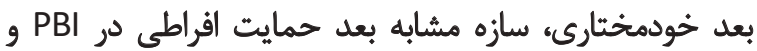
s-EMBU

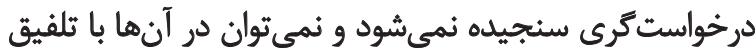

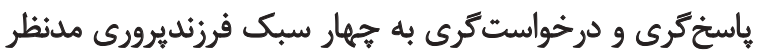

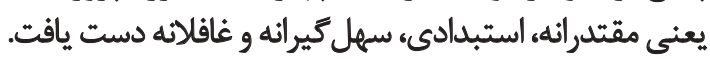

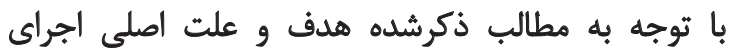

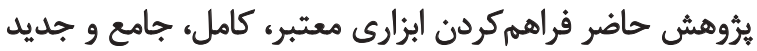

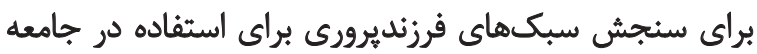

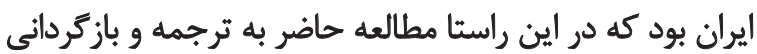

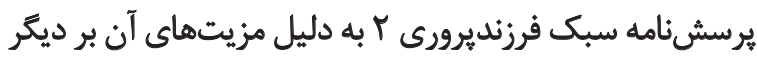

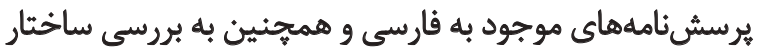

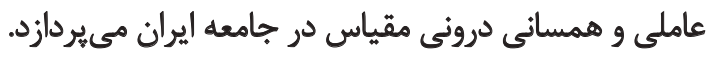

وروش

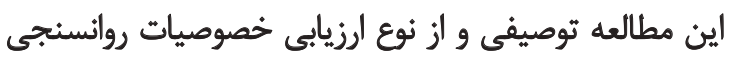

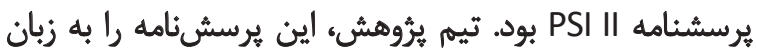

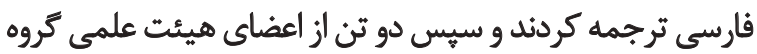

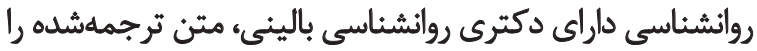

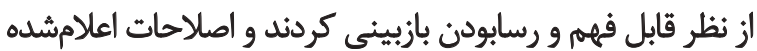

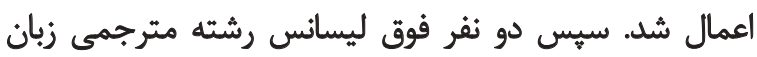

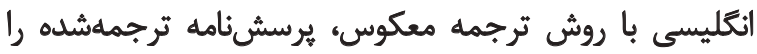

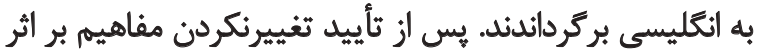

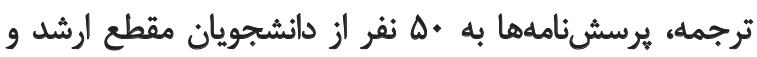

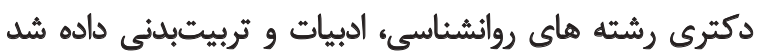

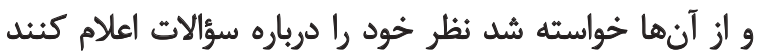

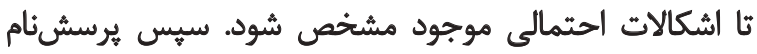

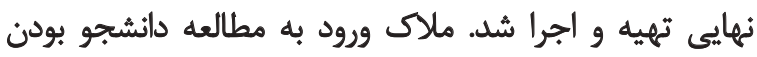

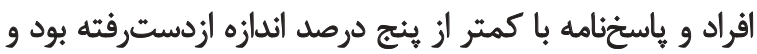

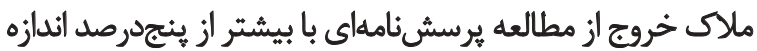

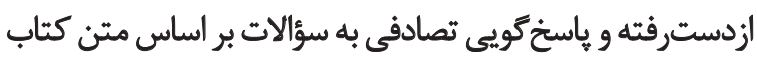

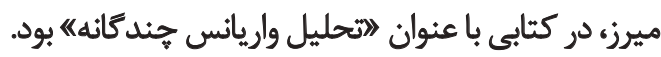

استبدادى، سهل كيرانه و غافلانه را با انتخاب يكى از بندها

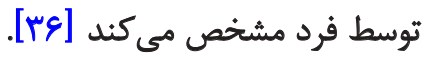

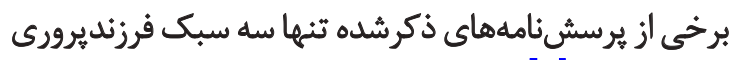

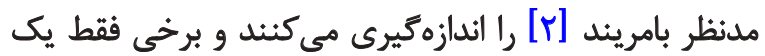

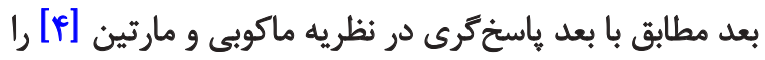

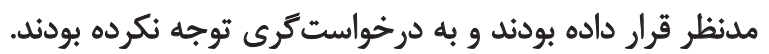

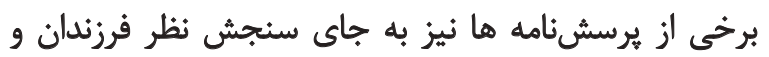

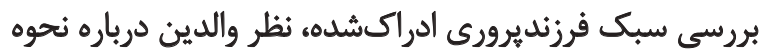

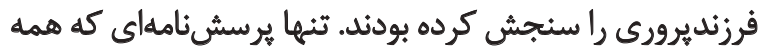

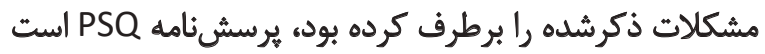

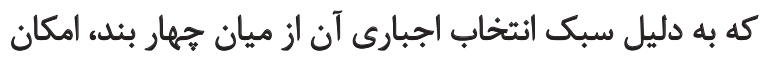

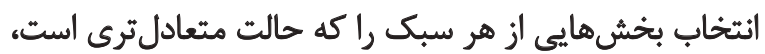

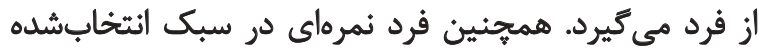

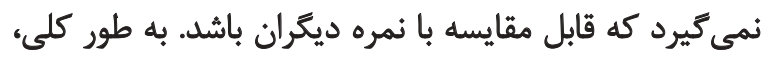

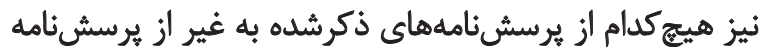

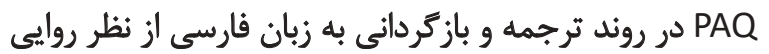

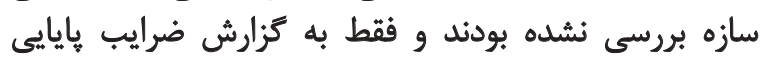

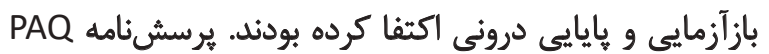

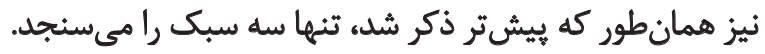

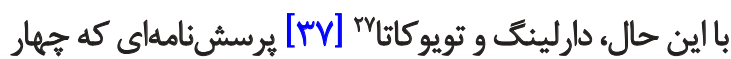

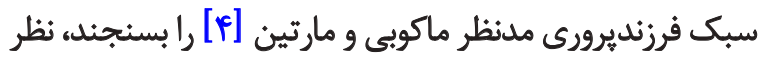

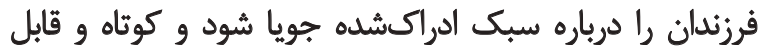

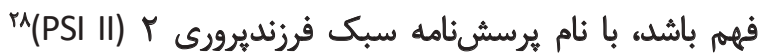

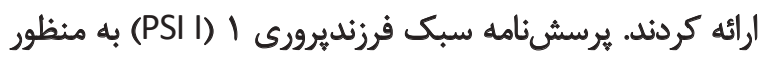

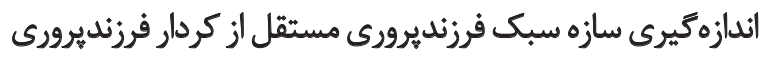

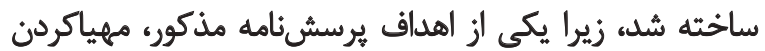

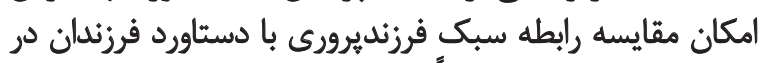

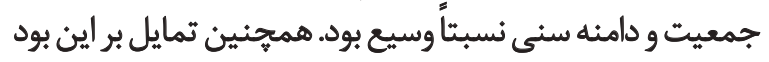

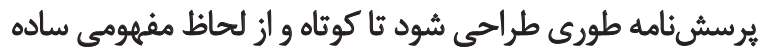

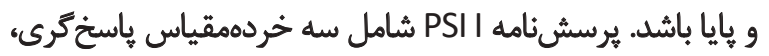

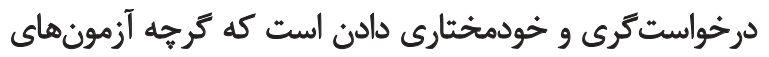

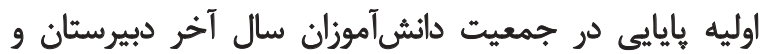

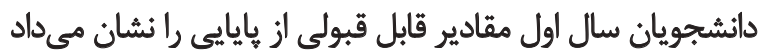

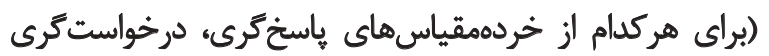

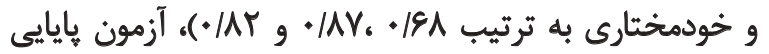

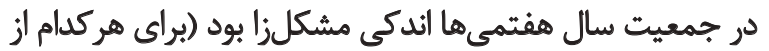

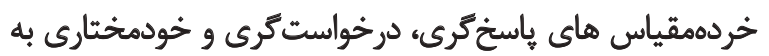

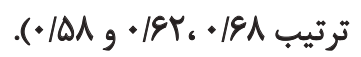

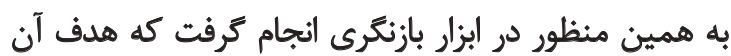

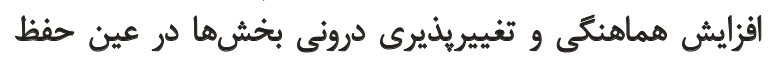

27. Darling \& Toyokawa

28. Parenting Style Inventory 


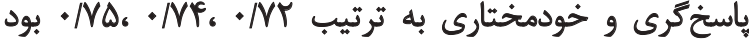
كه نشاندهنده يايايى خوبى استى

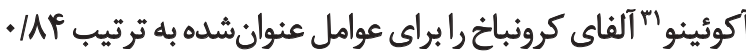

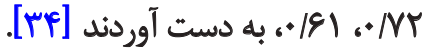

يافتهها

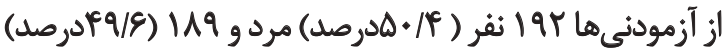

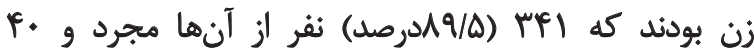

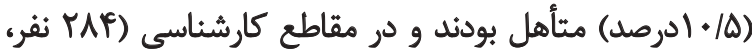

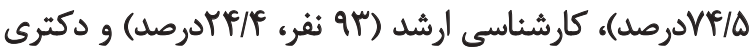

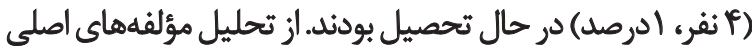

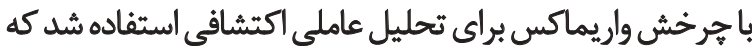

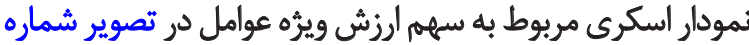

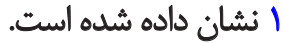

با اجراى تحليل عاملى نتايج با توجه به بيشفرض إِ ارزش

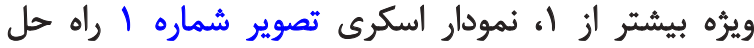

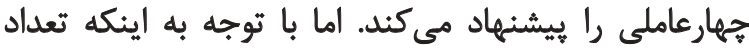

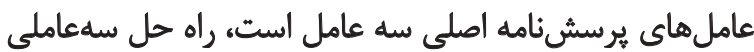

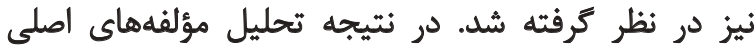

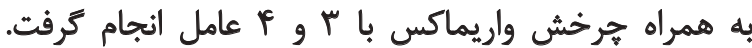

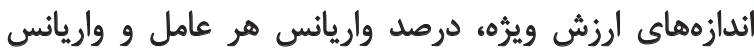

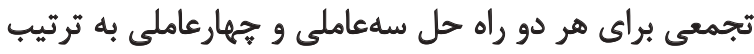

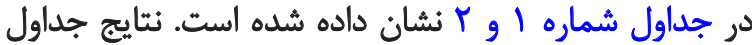

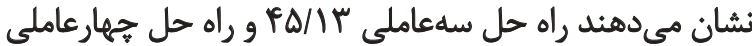

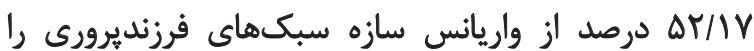

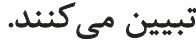
به منظور انتخاب مدل بهتر از ميان دو مدل سهعاملى و

31. Hardy, Bhattacharjee, Reed \& Aquin

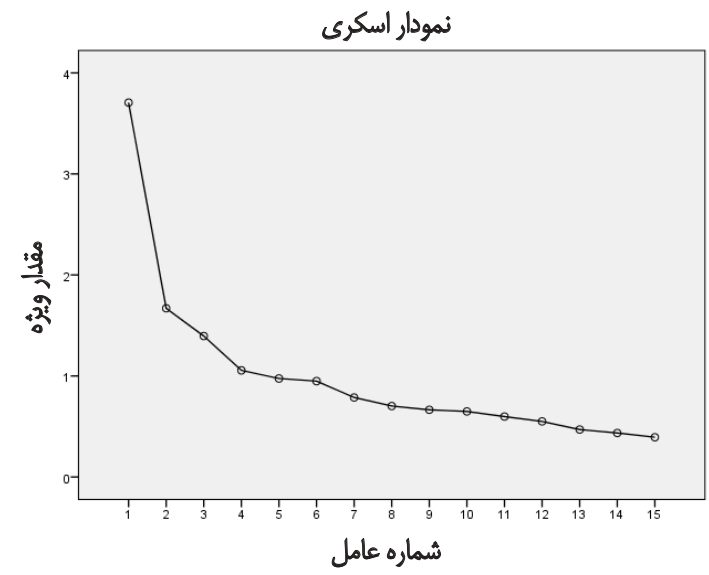

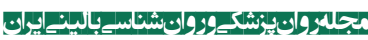

تصوير ا. نمودار اسكرى ميوبوط به الرزش ويرُّه تعيين شئه توسط عوامل

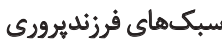

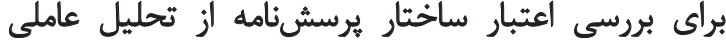

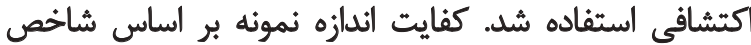

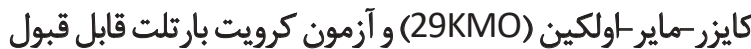

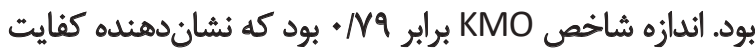

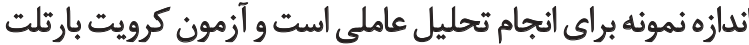

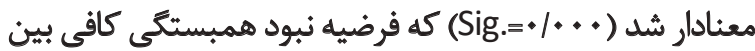

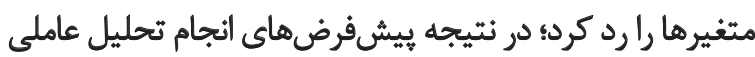

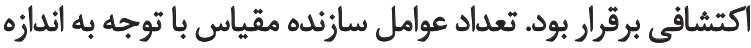

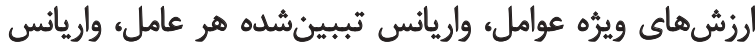

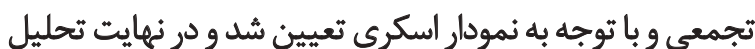

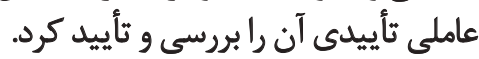

أزمودثىها

حجم نمونه يثوهش با استفاده از جدول موركان تعيين شد كه

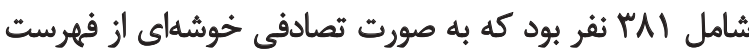

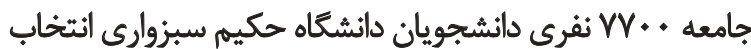

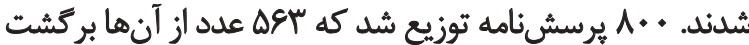

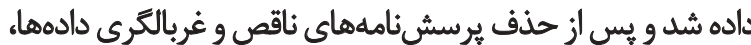

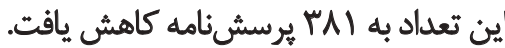

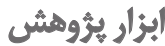

\section{برسشنامه سبك فرزنديرورى Y (PSI II)}

دارلينك و تويوكاوا براي اندازهيرى سبكهاي فرزنديروي سيري

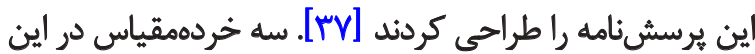

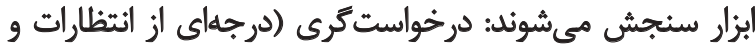

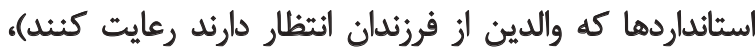

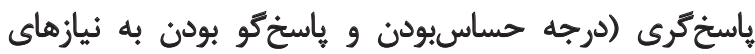

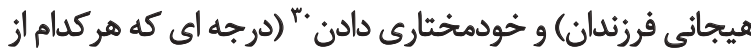

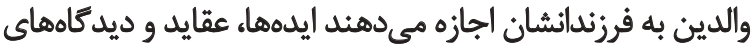
خود را كسترش دهند و آنها را تشويق مي كنيند). اين ابزار شامل ها بخش است كه روى يك مقياس ليكرت

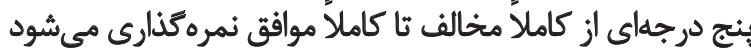

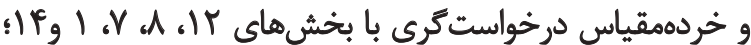

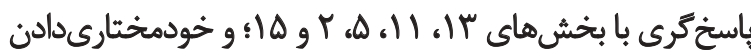

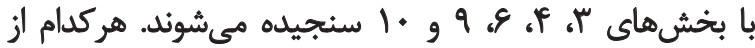

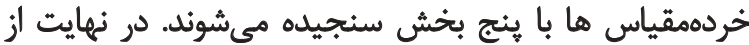

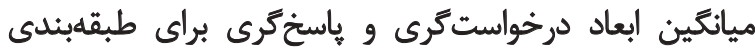

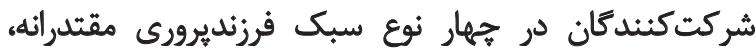

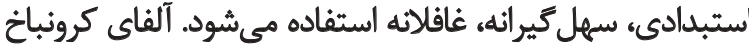

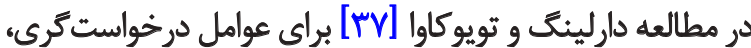

29. Kaiser-Meyer-Olkin

30. Autonomy Granting 
جدول ا. اندازههاي ارزش ويره، درصد واريانس هر عامل و درصد واريانس تجمعى راه حل سهعاملى

\begin{tabular}{|c|c|c|c|}
\hline درصد واريانس تجمعى & درصد واريانس & ارزش ويثه & عاملها \\
\hline 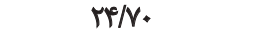 & $M F / V$ & $r / V$ & 1 \\
\hline$r \Delta / A r$ & $11 / 1 r$ & V/FV & r \\
\hline PQ/NT & q/r. & $1 / 1 \%$ & $r$ \\
\hline
\end{tabular}

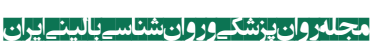

جدول r. أدازههاى ارزش ويزٔه، درصد واريانس هر عامل و درصد واريانس تجمعى راه حل جهارعاملى

\begin{tabular}{|c|c|c|c|}
\hline درصد واريانس تجمعى & درصد واريانس & ارزش ويزٔه & عاملها \\
\hline rive & MiN. & $r / N$ & 1 \\
\hline $\mathrm{ra/Ar}$ & $11 / 1 r$ & I/FV & r \\
\hline$r \Delta / 1 Y$ & q/r. & $1 / 19$ & r \\
\hline$\Delta T / I V$ & $V / \cdot r$ & $1 / .0$ & f \\
\hline
\end{tabular}

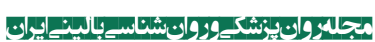

با توجه به برازش بهتر ساختار عاملى مدل سهعاملى با دادهها،

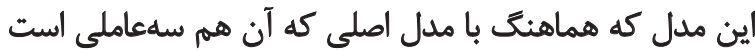

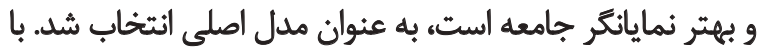

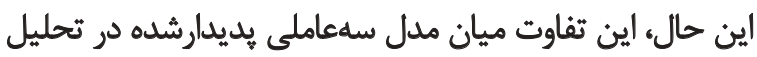

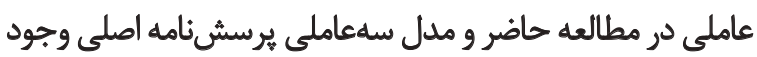

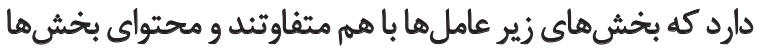

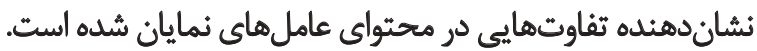

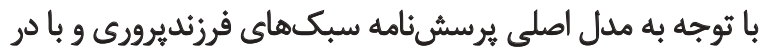

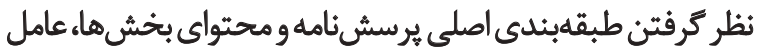

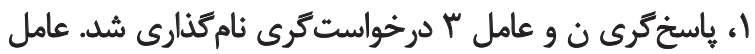

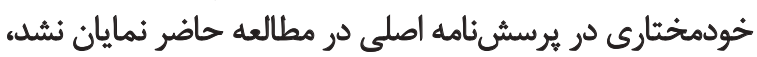

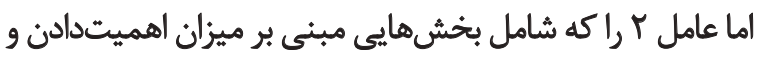

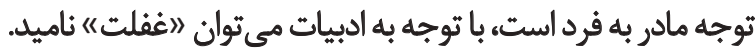

همساني درونى عوامل يرسشنامه با استفاده از آلفاي كرونباخ

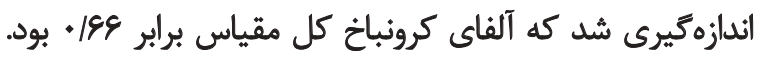

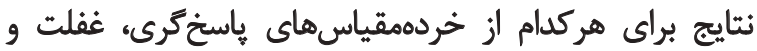

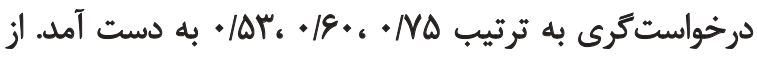

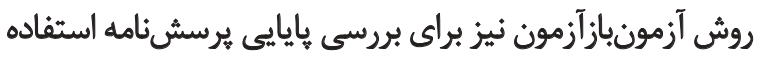

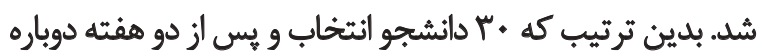

جهارعاملى از تحليل عاملي ثأييدى براي هر مدل استفاده شد.

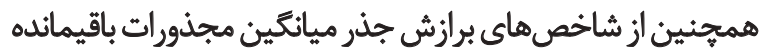
TrMSEA)

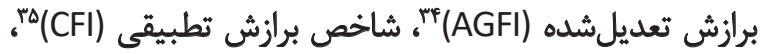

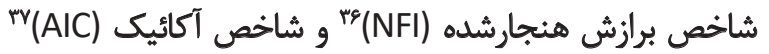

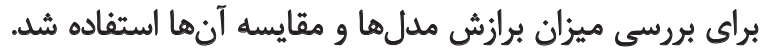

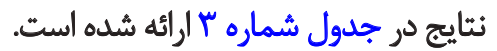

نتايج در جدول شماره ب نشان مى دهد كه همه شاخص هاى برازش براى هر دو مدل سهعاملى و جههارعاملى تثريباً برابرند،

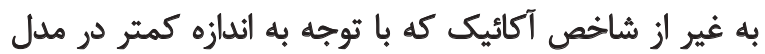

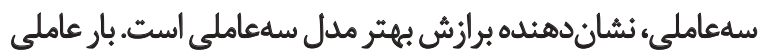

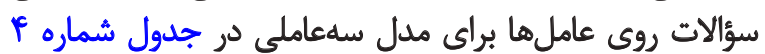

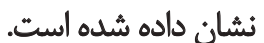

32. Root Mean Square Error of Approximation (RMSEA)

33. Goodness of Fit Index (GFI)

34. Adjusted Goodness of Fit Index (AGFI)

35. Comparative Fit Index (CFI)

36. Nonnormed Fit Index (NFI)

37. Akaike Information Criterion (AIC)

جدول ب. شاخص هاى برازش مدل هاى سهعاملى و جهارعاملي

\begin{tabular}{|c|c|c|c|c|c|c|c|c|}
\hline AIC & AGFI & GFI & NFI & CFI & RMSEA & $\mathbf{X}^{Y}$ & df & مدل \\
\hline$r+q / \lambda$. & $\cdot / \lambda F$ & $+/ M$ & .N. & - NA & .1 .9 & $r \vee \Delta / A$. & $M$ & سلهعاملى \\
\hline PFT/Rq & r & $\cdot / M$ & $\cdot N$ & - NO & .1 .9 & mVT/rq & 10 & جيهارعاملى \\
\hline
\end{tabular}


جدول f. بخش هاي يرسش نامه سبك فرزنديرورى و بارهاي عاملى آنها در مدل سهعاملى

\begin{tabular}{|c|c|c|c|}
\hline عامل r & عامل r & عامل I & بخشها \\
\hline & & . I89 & $r$ \\
\hline & & . IAF & 8 \\
\hline & & .190 & 1. \\
\hline & & .199 & 11 \\
\hline & & $\cdot / \Delta$ & ir \\
\hline & &.$/ 8 \Lambda$ & $\mathbb{1 r}$ \\
\hline & &.$/ 8$. & 10 \\
\hline & .199 & & $r$ \\
\hline &.$/ T V$ & & $r$ \\
\hline & $. / P \mid$ & & $\Delta$ \\
\hline & .198 & & $v$ \\
\hline$. / 1+9$ & & & 1 \\
\hline$\cdot / M^{\circ}$ & & & $\wedge$ \\
\hline .109 & & & 9 \\
\hline.$- / \Delta \mu^{\prime}$ & & & 19 \\
\hline
\end{tabular}

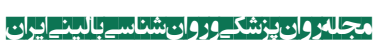

كرمى والدينى را از طريق ارتباط كلامى يا يشتيبانى از طريق

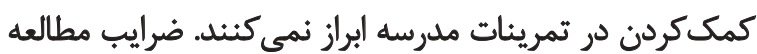

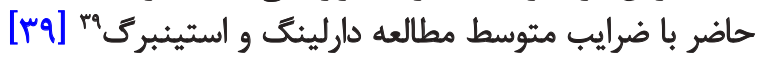

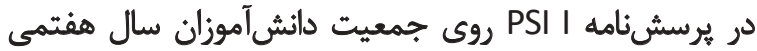

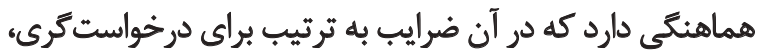

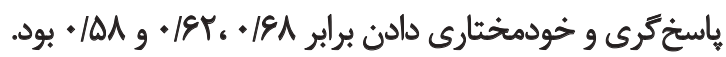

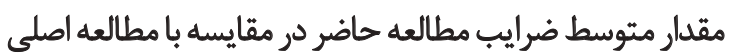

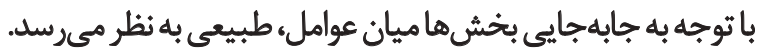

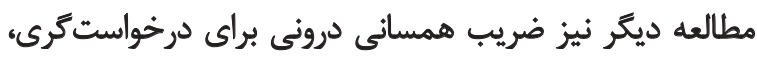

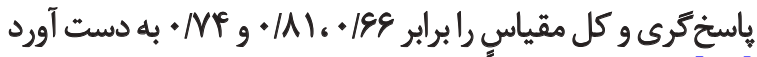

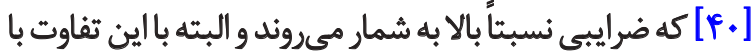

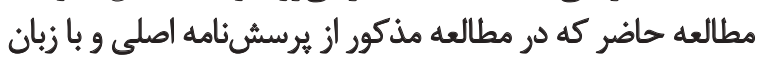

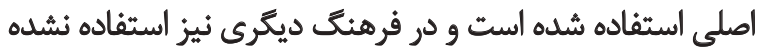

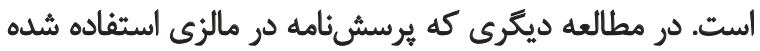

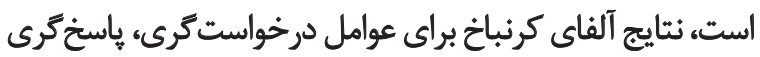

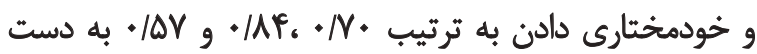

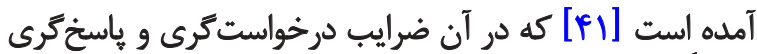

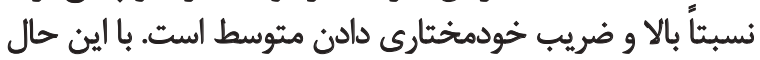

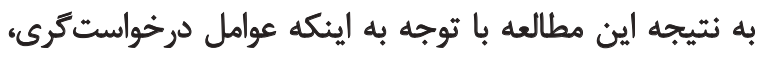

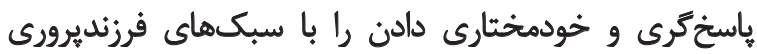

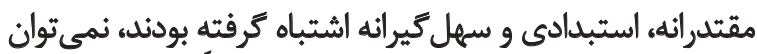

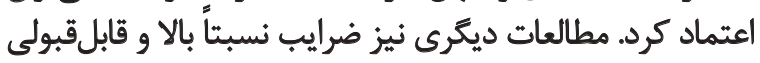

39. Darling \& Steinberg
به يرسش آنامه ياسخ دادند كه نتايج تحليل همبستكى نشان داد

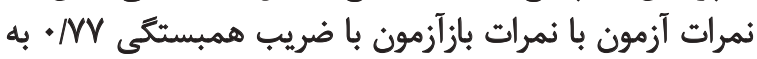

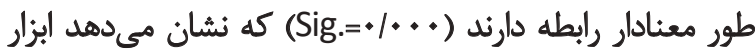

يايايي خوبى دارد.

ث

همانطور كه در قسمت نثايج كفته شد، پِّ از انجام تحليل

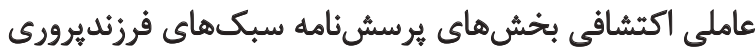

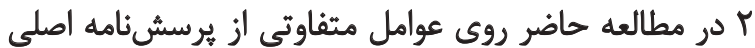

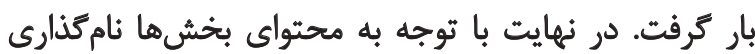

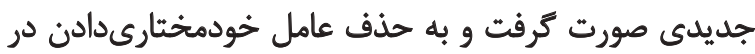

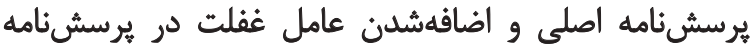
هنجاريابي شده منجر شد.

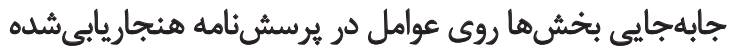

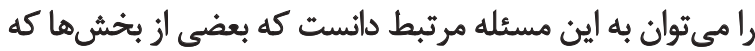

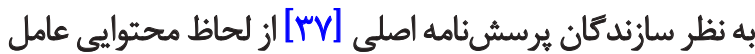

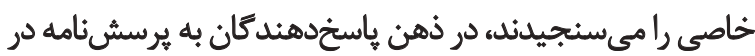

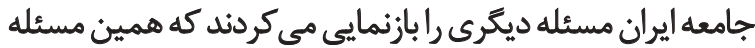

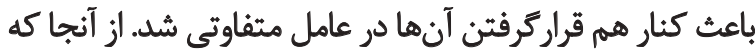

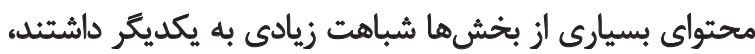

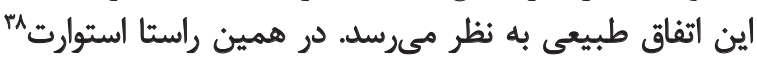

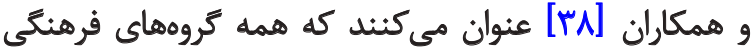

38. Stewart 


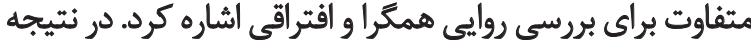

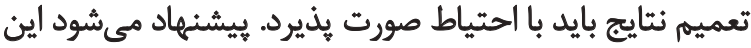

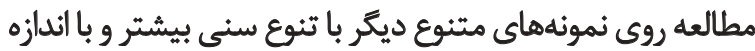

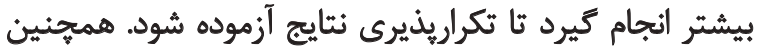

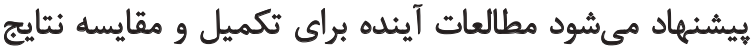

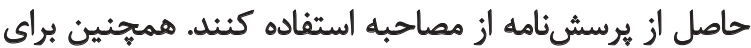

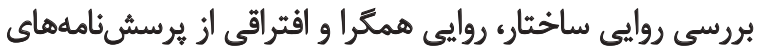

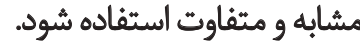

$$
\text { سياسئز الرى }
$$

بنا به اظهار نويسنده مسئول مقاله، تعارض منافع و حامى مالى

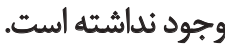

را براى عوامل يرسش ئامه سبكهاى فرزنديرورى كزّارش كرداند

[FY-FQ]

با وجود اينكه در بسيارى از مطالعات ضريب همسائى دروني

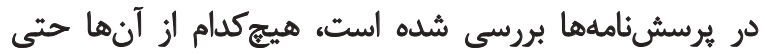

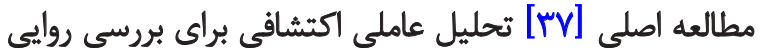

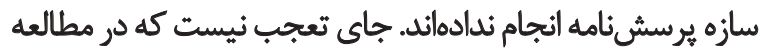

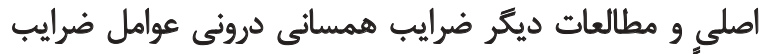

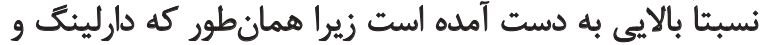

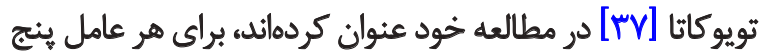

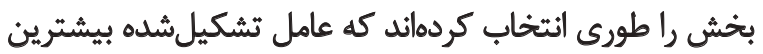

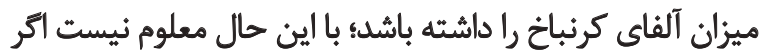

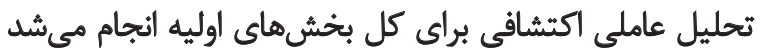
ساختارى مشابه ساختار نهايي ارائهشده نمايان مى بـ ئد يانه.

در مطالعه حاضر ضريب بايايايى آزمونبازآزمون مقدار نسبتاً

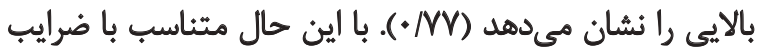

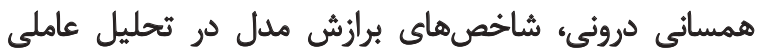

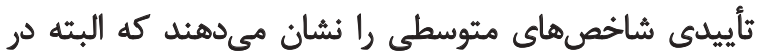

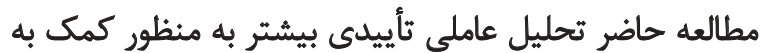

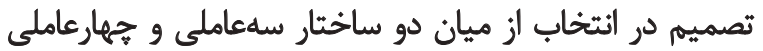
نمايانشده در تحليل عاملى اكتشافى استفاده شد.

تثيجليرى

مى توان گفت اين يرسش نامه يايايى نسبى داردو تحليل عوامل

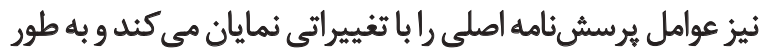

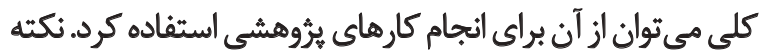

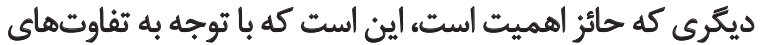

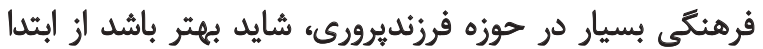

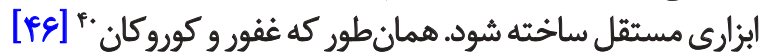

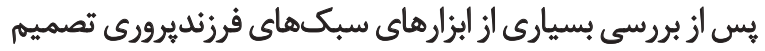

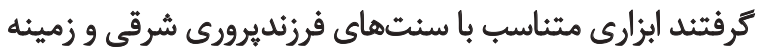

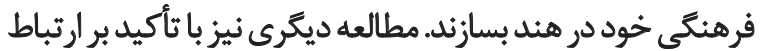

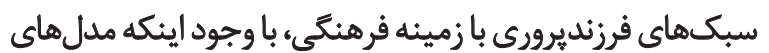

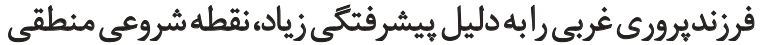

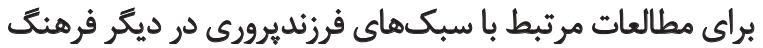

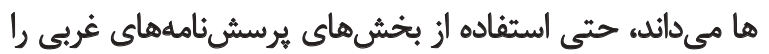

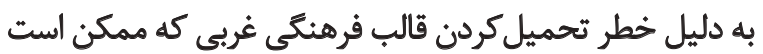

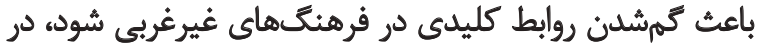

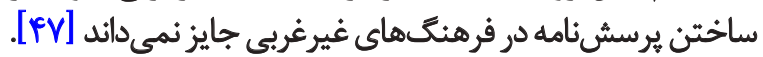
از محدوديتهاي مطالعه حاضر ميتوان به استفاده از ابزار

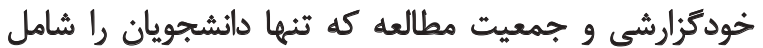

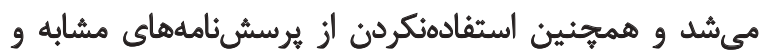

40. Gafor \& Kurukkan 


\section{References}

[1] Bradley RH, Caldwell BM. Caregiving and the regulation of child growth and development: Describing proximal aspects of caregiving systems. Developmental Review. 1995; 15(1):38-85. doi: 10.1006/drev.1995.1002

[2] Baumrind D. Current patterns of parental authority. Developmental Psychology. 1971; 4(1, Pt.2):1-103. doi: 10.1037/h0030372

[3] Dornbusch SM, Ritter PL, Leiderman PH, Roberts DF, Fraleigh MJ. The relation of parenting style to adolescent school performance. Child Development. 1987; 58(5):1244-57. doi: $10.2307 / 1130618$

[4] Maccoby EE, Martin JA. Socialization in the context of the family: Parent-child interaction. In: Hetherington EM, Mussen PH, editors. Handbook of Child Psychology, Socialization, Personality and Social Development. Hoboken, New Jersey: John Wiley \& Sons; 1983.

[5] Fan J, Zhang L. The role of perceived parenting styles in thinking styles. Learning and Individual Differences. 2014; 32:204-11. doi: 10.1016/j.lindif.2014.03.004

[6] Collins WA, Maccoby EE, Steinberg L, Hetherington EM, Bornstein $\mathrm{MH}$. Contemporary research on parenting: The case for nature and nurture. American Psychologist. 2000; 55(2):218-32. doi: 10.1037/0003-066x.55.2.218

[7] Steinberg L, Morris AS. Adolescent development. Journal of Cognitive Education and Psychology. 2001; 2(1):55-87. doi: 10.1891/194589501787383444

[8] Bornstein L, Bornstein MH. Parenting styles and child social development. $2^{\text {nd }}$ edition. New York: National Institute of Child Health and Human Development, 2014.

[9] Chen WW. The relations between perceived parenting styles and academic achievement in Hong Kong: The mediating role of students' goal orientations. Learning and Individual Differences. 2015; 37:48-54. doi: 10.1016/j.lindif.2014.11.021

[10] Kenney SR, Lac A, Hummer JF, Grimaldi EM, LaBrie JW. Pathways of parenting style on adolescents' college adjustment, academic achievement, and alcohol risk. Journal of College Student Retention: Research, Theory \& Practice. 2015; 17(2):186-203. doi: $10.1177 / 1521025115578232$

[11] ZAhedani Zz, Rezaee R, Yazdani Z, Bagheri S, Nabeiei P. The influence of parenting style on academic achievement and career path. Journal of Advances in Medical Education \& Professionalism. 2016; 4(3):130-4. PMCID: PMC4927255

[12] Doctoroff GL, Arnold DH. Doing homework together: The relation between parenting strategies, child engagement, and achievement. Journal of Applied Developmental Psychology. 2017; 48:103-13. doi: 10.1016/j.appdev.2017.01.001

[13] Perry RA, Daniels LA, Bell L, Magarey AM. Facilitators and barriers to the achievement of healthy lifestyle goals: Qualitative findings from Australian parents enrolled in the peach child weight management program. Journal of Nutrition Education and Behavior. 2017; 49(1):43-52. doi: 10.1016/i.jneb.2016.08.018

[14] Baldwin AL. Socialization and the parent-child relationship. Child Development. 1948; 19(3):127-36. doi: 10.2307/1125710

[15] García F, Gracia E. The indulgent parenting style and developmental outcomes in South European and Latin American countries. In: Selin H, editors. Parenting Across Cultures. Science
Across Cultures: The History of Non-Western Science. Berlin: Springer; 2014.

[16] Karim AR, Sharafat T, Mahmud AY. Cognitive emotion regulation in children as related to their parenting style, family type and gender. ournal of the Asiatic Society of Bangladesh, Science. 2014; 39(2):211-20. doi: 10.3329/jasbs.v39i2.17860

[17] Liew J, Kwok O, Chang Y-p, Chang BW, Yeh YC. Parental autonomy support predicts academic achievement through emotion-related self-regulation and adaptive skills in Chinese American adolescents. Asian American Journal of Psychology. 2014; 5(3):214-22. doi: 10.1037/a0034787

[18] Hasyim NNF, Naimie Z, Abuzaid RA, Halili SH, Siraj S. The relationship between authoritative parenting style and academic achievement. Paper presented at: The Annual International Conference on Management and Technology in Knowledge, Service, Tourism \& Hospitality. 23-24 August 2014; Jakarta, Indonesia.

[19] Sangawi H, Adams J, Reissland N. The impact of parenting styles on children developmental outcome: The role of academic self-concept as a mediator. International Journal of Psychology. 2016. doi: 10.1002/ijop.12380.

[20] Merz EC, Landry SH, Montroy JJ, Williams JM. Bidirectional associations between parental responsiveness and executive function during early childhood. Social Development. 2016; 26(3):591609. doi: 10.1111/sode.12204

[21] Soenens B, Deci EL, Vansteenkiste M. How Parents Contribute to Children's Psychological Health: The Critical Role of Psychological Need Support. In: Wehmeyer M, Shogren K, Little T, Lopez S, editors. Development of Self-Determination Through the Life-Course. Berlin: Springer; 2017.

[22] Baumrind D. The development of instrumental competence through socialization. In: Pick A, editor. Minnesota Symposia on Child Psychology. Minneapolis: University of Minnesota Press; 1973.

[23] Parker G, Tupling H, Brown L. A parental bonding instrument. British Journal of Medical Psychology. 1979; 52(1):1-10.

[24] Buri JR. Parental authority questionnaire. Journal of Personality Assessment. 1991; 57(1):110-9. doi: 10.1207/s15327752jpa5701_13

[25] Robinson CC, Mandleco B, Olsen SF, Hart CH. Authoritative, authoritarian, and permissive parenting practices: Developmen of a new measure. Psychological Reports. 1995; 77(3):819-30. doi: 10.2466/pr0.1995.77.3.819

[26] McClun LA, Merrell KW. Relationship of perceived parenting styles, locus of control orientation, and self-concept among junior high age students. Psychology in the Schools. 1998; 35(4):381-90 doi: 10.1002/(sici)1520-6807(199810)35:4<381::aid-pits9>3.3.co;2-j

[27] Arrindell WA, Sanavio E, Aguilar G, Sica C, Hatzichristou C, Eisemann $\mathrm{M}$, et al. The development of a short form of the EMBU: Its appraisal with students in Greece, Guatemala, Hungary and Italy. Personality and Individual Differences. 1999; 27(4):613-28. doi: 10.1016/s0191-8869(98)00192-5

[28] Zeinali A, Vahdat R, Garadingeh K. [The Relationship Between Parenting Style and Addiction Susceptibility in Children (Persian)]. Journal of Family Research. 2010; 6(23):335-52.

[29] Moazen T, Aghei A, Golparvar M. [Predicted Students Attachment styles based on parents parenting styles (Persian)]. Journal of Educational Sciences. 2014; 7(25):87-99. 
[30] Danesh E, Rezabakhsh H, Bahmani Z, Saliminia N. [Relationship between parenting styles and sexual self esteem and its' components in university students (Persian.)]. Journal of Applied Psychology. 2011; 5(3): 39-55.

[31] Ghannadi F, Abdollahi MH. [The relationship between perceptions of parental behavior and early maladaptive schemas (Persian)]. Clinical Psychology Studies. 2014; 4(16):129-51.

[32] Ejei J, Lavasani M G, Malahmadi E, Khezri M. [The relationship between parenting styles and academic achievement through the mediating influences of achievement goals and academic (Persian)]. Journal of Psychology. 2011; 15(3):284-301.

[33] Alizadeh H, Andries C. Interaction of parenting styles and attention deficit hyperactivity disorder in Iranian parents. Child \& Family Behavior Therapy. 2002; 24(3):37-52. doi: 10.1300/ j019v24n03_03

[34] Purabdoli M, Kadivar, P, Homayuni A. [To investigate the relationship between motherparenting styles and their children's perception (Persian)]. Knowledge \& Research in Applied Psychology. 2008; 10(37):107-28.

[35] Hassani M, Fathi-Ashtiani A, Rasoolzadeh-Tabatabaei SK. [A comparison of early maladaptive schemas and perceived parental rearing behaviors in couples with problem-focused and emotionfocused coping style (Persian.)]. International Journal of Behavioral Sciences. 2012; 6(3):9-10.

[36] Neumeister KLS, Finch H. Perfectionism in high-ability students: Relational precursors and influences on achievement motivation. Gifted Child Quarterly. 2006; 50(3):238-51. doi: $10.1177 / 001698620605000304$

[37] Darling N, Toyokawa T. Construction and validation of the parenting style inventory II (PSI-II). Pennsylvania: The Pennsylvania State University; 1997.

[38] Stewart SM, Bond MH, Zaman RM, McBride-Chang C, Rao $\mathrm{N}$, Ho L, et al. Functional parenting in Pakistan. International Journal of Behavioral Development. 1999; 23(3):747-70. doi; $10.1080 / 016502599383784$

[39] Darling N, Steinberg L. Parenting style as context: An integrative model. Psychological Bulletin. 1993; 113(3):487-96. doi: 10.1037//0033-2909.113.3.487

[40] Vargas C. [Perceived parenting styles influence on contraceptive use among adolescents: a retrospective study on young adults'behavior] [MSc. thesis]. Gainesville, Florida: University of Florida; 2010.

[41] Ying GL. [Perceived parenting style and youth suicidality in Malaysia: Department of Psychology and Counseling, Faculty of Arts and Social Science] [MSc. thesis]. Petaling Jaya: Universiti Tunku Abdul Rahman; 2013.

[42] Carlo G, McGinley M, Hayes R, Batenhorst C, Wilkinson J. Parenting styles or practices? Parenting, sympathy, and prosocial behaviors among adolescents. The Journal of Genetic Psychology. 2007; 168(2):147-76. doi: 10.3200/gntp.168.2.147-176

[43] Hardy SA, Bhattacharjee A, Reed II A, Aquino K. Moral identity and psychological distance: The case of adolescent parental socialization. Journal of Adolescence. 2010; 33(1):111-23. doi: 10.1016/j.adolescence.2009.04.008

[44] Bastaits K, Ponnet K, Mortelmans D. Parenting of divorced fathers and the association with children's self-esteem. Journal of Youth and Adolescence. 2012; 41(12):1643-56. doi: 10.1007/ s10964-012-9783-6

[45] Lam CB, Solmeyer AR, McHale SM. Sibling relationships and empathy across the transition to adolescence. Journal of Youth and Adolescence. 2012; 41(12):1657-70. doi: 10.1007/s10964-0129781-8

[46] Abdul Gafor K, Kurukkan A. Construction and Validation of Scale of Parenting Style. Guru Journal of Behavioral and Social Sciences. 2014; 2(4):315-23.

[47] Batool SS, Mumtaz AN. Development and Validation of Parenting Style Scale. Pakistan Journal of Psychological Research. 2015; 30(2):225-48. 